\title{
A Nonsmooth Dynamics Perspective on State Observer Design for Mechanical Systems with Unilateral Constraints
}

\author{
Pascal V. Preiswerk, Remco I. Leine \\ Institute for Nonlinear Mechanics \\ University of Stuttgart \\ Pfaffenwaldring 9, 70569 Stuttgart, Germany \\ [preiswerk, leine]@inm.uni-stuttgart.de
}

\begin{abstract}
One of the main difficulties in the state observer design for impulsive mechanical systems is the so-called peaking phenomenon: even for an arbitrarily small pre-impact estimation error, a slight mismatch between the impact time instants of the observer and the observed system can lead to large post-impact estimation error. Therefore, Lyapunov's stability theorems cannot be directly applied. For linear mechanical systems with unilateral constraints, we propose to take a Nonsmooth Dynamics perspective on the problem, which allows to sidestep the main difficulties by transforming and approximating the original continuous-time system by a discrete linear complementarity system through the use of the Schatzman-Paoli scheme. The discretization acts as a regularization, i.e. the impacts take place over two consecutive time steps. Furthermore, it involves force and impact laws on position-level with the favorable property of maximal monotonicity. Finally, a passivity-based observer design for discrete linear complementarity systems can be applied.
\end{abstract}

Keywords: Nonsmooth systems, linear complementarity systems, impacts, unilateral constraints, peaking phenomenon.

\section{INTRODUCTION}

An important aspect of the state observer design for impulsive mechanical systems is whether or not the impact time instants, where state jumps occur, are known. Most proposed observers assume that these impact time instants can directly be extracted from measurements, for example by measuring all relevant positions in a system where the impact time instants are position dependent $[1,2]$ or by directly measuring contact [3]. This allows for the design of a state observer that exhibits impacts (or state jumps) that occur at the same time instants as in the observed system. Under a maximal monotone impact law, it is then possible to construct a Lyapunov function which does not increase over impacts, and with which asymptotic stability of the error dynamics (i.e. the time evolution of the difference between the estimated state and the actual state) can be shown.

Only few attempts have been made to design state observers in the case of unknown impact time instants, such that the corresponding state jumps of the observed system and the state observer do not coincide. One of the main difficulties in that case is the peaking phenomenon: even for an arbitrarily small pre-impact estimation error, a slight mismatch in the impact time instants can lead to a large post-impact velocity error caused by velocity jumps. This makes it difficult to show asymptotic stability of the estimation error dynamics using Lyapunov's stability theory. In fact, due to the peaking phenomenon, the estimation error dynamics is not Lyapunov stable by definition. One approach for such systems is to find a state transformation from the original system into a new system without state jumps [4, 5], for which conventional state observer techniques can be applied. However, such a transformation does not always exist and is in general difficult to find. In this paper, we aim at sidestepping the main difficulties in the observer design for impulsive mechanical systems by first discretizing the continuous-time problem using the Schatzman-Paoli scheme, in which force and impact laws are formulated on position level with the favorable property of maximal monotonicity. 


\section{CONTINUOUS-TIME PROBLEM}

We consider a linear mechanical system which is subjected to unilateral constraints and a timedependent external forcing. Let $\mathbf{q}(t)$ be the generalized coordinates and $\mathbf{u}(t)$ be the corresponding generalized velocities. In general, $\mathbf{u}(t)$ is assumed to be a function of bounded variation, leading to absolutely continuous $\mathbf{q}(t)$. The non-impulsive part of the dynamics is described by

$$
\begin{aligned}
& \dot{\mathbf{q}}=\mathbf{u}, \\
& \mathbf{M} \dot{\mathbf{u}}+\mathbf{K q}+\mathbf{D u}=\mathbf{W} \boldsymbol{\lambda}+\mathbf{f}(t),
\end{aligned}
$$

where the the mass matrix $\mathbf{M}=\mathbf{M}^{\top} \succ 0$, the stiffness matrix $\mathbf{K}=\mathbf{K}^{\top} \succ 0$ and the damping matrix $\mathbf{D} \succ 0$ are assumed to be constant and positive definite. The unilateral constraints are described by linear inequality conditions $\mathbf{g}(\mathbf{q})=\mathbf{W}^{\top} \mathbf{q} \geq 0$, leading to the non-impulsive constraint forces $\boldsymbol{\lambda}$. The generalized force directions, given by the columns of $\mathbf{W}=\left(\frac{\partial \mathbf{g}}{\partial \mathbf{q}}\right)^{\top}$, are assumed to be constant and linearly independent (such that $\mathbf{W}$ has full rank). Furthermore, the system is excited by a bounded, time-dependent external forcing $\mathbf{f}(t)$. Note that the equations of motion (1) by themselves do not allow for discontinuities in the generalized velocities $\mathbf{u}$. In order to describe velocity jumps due to impacts, the impulsive part of the dynamics, i.e. the impact equations, are given by

$$
\mathbf{M}\left(\mathbf{u}^{+}-\mathbf{u}^{-}\right)=\mathbf{W} \mathbf{\Lambda},
$$

where $\mathbf{u}^{+}(t)$ and $\mathbf{u}^{-}(t)$ denote the left and right limit of $\mathbf{u}$ at time $t$ and $\boldsymbol{\Lambda}$ are the impulsive constraint forces. For the components of the constraint forces $\boldsymbol{\lambda}$ and $\boldsymbol{\Lambda}$ we assume Signorini's law on position level

$$
\begin{aligned}
& 0 \leq \mathbf{g} \perp \boldsymbol{\lambda} \geq 0, \\
& 0 \leq \mathbf{g} \perp \boldsymbol{\Lambda} \geq 0,
\end{aligned}
$$

where we used the notation $\mathbf{g} \geq 0$ to express the non-negativity of every component $g_{i} \geq 0 \forall i$ and the notation $\mathbf{g} \perp \boldsymbol{\lambda}$ to express the orthogonality $\mathbf{g}^{\top} \boldsymbol{\lambda}=0$. Hence, (3a) is equivalent to $g_{i} \geq 0, \lambda_{i} \geq$ $0, g_{i} \lambda_{i}=0$ for all $i$ and is referred to as an inequality complementarity condition. The constraint forces can alternatively be formulated on position-switched velocity level [6]: with $\boldsymbol{\gamma}=\mathbf{W}^{\top} \mathbf{u}$, the laws (3) are equivalent to the component-wise law

$$
\begin{array}{ll}
g_{i}(\mathbf{q})=0: 0 \leq \gamma_{i} \perp \lambda_{i} \geq 0, & g_{i}(\mathbf{q})>0: \lambda_{i}=0, \\
g_{i}(\mathbf{q})=0: 0 \leq \gamma_{i} \perp \Lambda_{i} \geq 0, & g_{i}(\mathbf{q})>0: \Lambda_{i}=0 .
\end{array}
$$

In addition to the force laws (4), an impact law has to be specified for a full description of the dynamics. Instantaneous impact laws directly relate post-impact relative velocities to pre-impact relative velocities and, in consistency with (4), are formulated on velocity level. Here, we will make use of a generalized Newtonian impact law [7], which is written component-wise as

$$
g_{i}(\mathbf{q})=0: 0 \leq \xi_{i} \perp \Lambda_{i} \geq 0, \quad g_{i}(\mathbf{q})>0: \Lambda_{i}=0,
$$

with the kinematic variables $\xi_{i}:=\gamma_{i}^{+}+\varepsilon_{i} \gamma_{i}^{-}$and given coefficients of restitution $\varepsilon_{i} \in[0,1]$.

In view of the time discretization, it is convenient to merge (1) and (2) in a compact formulation containing both the non-impulsive and the impulsive dynamics. This leads to an equality of measures of the form $[8,9]$

$$
\begin{aligned}
& \mathrm{d} \mathbf{q}=\mathbf{u d} t \\
& \mathbf{M} \mathrm{d} \mathbf{u}+(\mathbf{K q}+\mathbf{D u}-\mathbf{f}(t)) \mathrm{d} t=\mathbf{W} \mathrm{d} \mathbf{P},
\end{aligned}
$$

where dq is the so-called differential measure of the generalized coordinates $\mathbf{q}$. Similarly, du= $\dot{\mathbf{u}} \mathrm{d} t+\left(\mathbf{u}^{+}-\mathbf{u}^{-}\right) \mathrm{d} \eta$ is the differential measure of the generalized velocities, allowing for discontinuities in the generalized velocities $\mathbf{u}$. Herein, $\mathrm{d} \eta$ is an atomic measure, being the sum of Dirac point measures [8]. Furthermore, $\mathrm{d} \mathbf{P}=\boldsymbol{\lambda} \mathrm{d} t+\boldsymbol{\Lambda} \mathrm{d} \eta$ is the differential contact effort measure. 
The force laws (4) and the impact law (5) can be gathered in a description of measures

$$
g_{i}(\mathbf{q})=0: 0 \leq \xi_{i} \perp \int_{\mathscr{I}} \mathrm{d} P_{i} \geq 0, \quad g_{i}(\mathbf{q})>0: \int_{\mathscr{I}} \mathrm{d} P_{i}=0,
$$

where the sign (or nullity) of $g_{i}$ and $\xi_{i}$ is assumed to be constant during the interval $\mathscr{I}$ (e.g. a short time step as used in a time discretization). For brevity, (7) is usually written as

$$
g_{i}(\mathbf{q})=0: 0 \leq \xi_{i} \perp \mathrm{d} P_{i} \geq 0, \quad g_{i}(\mathbf{q})>0: \mathrm{d} P_{i}=0,
$$

refraining from referring to the assumption on $\mathscr{I}$.

\subsection{DISCRETE-TIME PROBLEM}

In the following we pursue an approach, where we first discretize the dynamics and then design a state observer for the discrete (and therefore approximate) system. As explained in the Introduction, this alleviates the problem of state jumps in the observer design.

Here, we will make use of the scheme of Schatzman and Paoli $[10,11]$. This scheme involves an impact law on position level, and was originally motivated by the fact that it allows for a rigorous convergence proof (which is not given for other, more widely used schemes such as the Moreau scheme [9]). The reason for choosing this scheme is the fact that its direct formulation of the contact/impact law on position level gives access to the maximal monotonicity property. However, the practical application of this scheme is restricted to mechanical systems with frictionless unilateral constraints which are decoupled such that $\mathbf{w}_{i}^{\top} \mathbf{M}^{-1} \mathbf{w}_{j}=0$ for $i \neq j$, where $\mathbf{w}_{i}=\frac{\partial g_{i}}{\partial \mathbf{q}}$. For system (6), the Schatzman-Paoli discretization scheme can be written as

$$
\begin{aligned}
& \mathbf{q}_{k+1}=\mathbf{q}_{k}+\Delta t \mathbf{u}_{k+1}, \\
& \mathbf{M}\left(\mathbf{u}_{k+1}-\mathbf{u}_{k}\right)+\left(\mathbf{K} \mathbf{q}_{k}+\mathbf{D} \mathbf{u}_{k}-\mathbf{f}_{k}\right) \Delta t=\mathbf{W P}_{k},
\end{aligned}
$$

together with

$$
\begin{aligned}
\boldsymbol{\xi}_{k} & :=\mathbf{g}_{k+1}+\varepsilon \mathbf{g}_{k-1}, \\
0 & \leq \boldsymbol{\xi}_{k} \perp \mathbf{P}_{k} \geq 0 .
\end{aligned}
$$

Therein, $\Delta t$ is the (constant) time step and variables being evaluated (or approximated) at the discrete times $t=t_{k}:=k \Delta t$ are referred to with an index $k$, e.g. $\mathbf{q}_{k}:=\mathbf{q}\left(t_{k}\right)$. Likewise, the discrete contact distance is $\mathbf{g}_{k}=\mathbf{W}^{\top} \mathbf{q}_{k}$ and the corresponding discrete contact velocity is $\boldsymbol{\gamma}_{k}=\mathbf{W}^{\top} \mathbf{u}_{k}$. To keep it simple, we will assume all coefficients of restitution $\varepsilon_{i}=\varepsilon$ to be equal. An important aspect of the Schatzman-Paoli scheme that the discrete impact law (10), i.e. $0 \leq \mathbf{g}_{k+1}+\varepsilon \mathbf{g}_{k-1} \perp \mathbf{P}_{k} \geq 0$, is formulated on position level. Other discretization schemes, which directly discretize the combined contact/impact law (8) on velocity level, require the introduction of an index set, indicating which contacts are closed at a given time instant. This is not the case for the Schatzman-Paoli scheme, since the discrete impact law (10) is not a direct discretization of (8). To understand its meaning, let $\boldsymbol{\xi}_{k}$ vanish over two consecutive time steps, i.e. $\boldsymbol{\xi}_{k-1}=\boldsymbol{\xi}_{k}=\mathbf{0}$. It then follows from the definition of $\boldsymbol{\xi}_{k}$ in (10) that

$$
\begin{aligned}
\frac{\boldsymbol{\xi}_{k}-\boldsymbol{\xi}_{k-1}}{\Delta t} & =\frac{\mathbf{g}_{k+1}-\mathbf{g}_{k}}{\Delta t}+\varepsilon \frac{\mathbf{g}_{k-1}-\mathbf{g}_{k-2}}{\Delta t}=\mathbf{W}^{\top}\left(\frac{\mathbf{q}_{k+1}-\mathbf{q}_{k}}{\Delta t}+\varepsilon \frac{\mathbf{q}_{k-1}-\mathbf{q}_{k-2}}{\Delta t}\right) \\
& =\mathbf{W}^{\top}\left(\mathbf{u}_{k+1}+\varepsilon \mathbf{u}_{k-1}\right)=\boldsymbol{\gamma}_{k+1}+\varepsilon \boldsymbol{\gamma}_{k-1}=\mathbf{0} .
\end{aligned}
$$

The last equality, $\boldsymbol{\gamma}_{k+1}+\varepsilon \boldsymbol{\gamma}_{k-1}=\mathbf{0}$, shows that Newton's impact law is fulfilled in a discretized sense over two time steps. Velocity jumps that occur instantaneously in continuous time take place over an interval of two time steps in the discretization, which can be seen as a regularization. Interestingly, the discretized system (9), (10) can be rewritten as what is known as a discrete 
linear complementarity system (LCS, as introduced in $[12,13])$. Indeed, by introducing the state $\mathbf{x}_{k}:=\left(\begin{array}{ll}\mathbf{q}_{k}^{\top} & \mathbf{u}_{k}^{\top}\end{array}\right)^{\top}$, equations (9) can be written as

$$
\left(\begin{array}{cc}
\mathbf{I} & -\Delta t \mathbf{I} \\
\mathbf{0} & \mathbf{M}
\end{array}\right) \mathbf{x}_{k+1}=\left(\begin{array}{cc}
\mathbf{I} & \mathbf{0} \\
-\Delta t \mathbf{K} & \mathbf{M}-\Delta t \mathbf{D}
\end{array}\right) \mathbf{x}_{k}+\left(\begin{array}{c}
\mathbf{0} \\
\mathbf{W}
\end{array}\right) \mathbf{P}_{k}+\left(\begin{array}{c}
\mathbf{0} \\
\Delta t \mathbf{I}
\end{array}\right) \mathbf{f}_{k} .
$$

Note that we are writing variables and matrices related to the state-space description without serifs, whereas in our original description of the mechanical system we are using serifs (therefore, variables denoted by the same letter, are assigned a different meaning depending on whether they are written with or without serifs). After inverting the matrix on the left hand side, an update rule for the state is obtained as

$$
\mathbf{x}_{k+1}=\left(\begin{array}{cc}
\mathbf{I} & \Delta t \mathbf{M}^{-1} \\
\mathbf{0} & \mathbf{M}^{-1}
\end{array}\right)\left[\left(\begin{array}{cc}
\mathbf{I} & \mathbf{0} \\
-\Delta t \mathbf{K} & \mathbf{M}-\Delta t \mathbf{D}
\end{array}\right) \mathbf{x}_{k}+\left(\begin{array}{c}
\mathbf{0} \\
\mathbf{W}
\end{array}\right) \mathbf{P}_{k}+\left(\begin{array}{c}
\mathbf{0} \\
\Delta t \mathbf{I}
\end{array}\right) \mathbf{f}_{k}\right]
$$

where $\mathbf{0}$ denotes a zero matrix of appropriate dimensions. Finally, after simple matrix multiplications we arrive at

$$
\mathbf{x}_{k+1}=\mathbf{A} \mathbf{x}_{k}+\mathbf{B} \mathbf{P}_{k}+\mathbf{E} \mathbf{f}_{k},
$$

with the corresponding system matrices $\mathbf{A}, \mathbf{B}$ and $\mathbf{E}$ given by

$$
\mathbf{A}=\left(\begin{array}{cc}
\mathbf{I}-\Delta t^{2} \mathbf{M}^{-1} \mathbf{K} & \Delta t\left(\mathbf{I}-\Delta t \mathbf{M}^{-1} \mathbf{D}\right) \\
-\Delta t \mathbf{M}^{-1} \mathbf{K} & \mathbf{I}-\Delta t \mathbf{M}^{-1} \mathbf{D}
\end{array}\right), \quad \mathbf{B}=\left(\begin{array}{c}
\Delta t \mathbf{M}^{-1} \mathbf{W} \\
\mathbf{M}^{-1} \mathbf{W}
\end{array}\right), \quad \mathbf{E}=\left(\begin{array}{c}
\Delta t^{2} \mathbf{M}^{-1} \\
\Delta t \mathbf{M}^{-1}
\end{array}\right)
$$

The discrete contact/impact law (10) can also be written in the state variables. By using the contact distance $\mathbf{g}_{k}=\mathbf{W}^{\top} \mathbf{q}_{k}$ and the first equation of (9) in (10) we have

$$
\begin{aligned}
& \boldsymbol{\xi}_{k}=\mathbf{W}^{\top}\left(\mathbf{q}_{k+1}+\varepsilon \mathbf{q}_{k-1}\right)=\mathbf{W}^{\top}\left(\mathbf{q}_{k+1}+\varepsilon\left(\mathbf{q}_{k}-\Delta t \mathbf{u}_{k}\right)\right) \\
& =\left(\begin{array}{ll}
\mathbf{W}^{\top} & \mathbf{0}
\end{array}\right) \mathbf{x}_{k+1}+\varepsilon\left(\mathbf{W}^{\top} \quad-\Delta t \mathbf{W}^{\top}\right) \mathbf{x}_{k}
\end{aligned}
$$

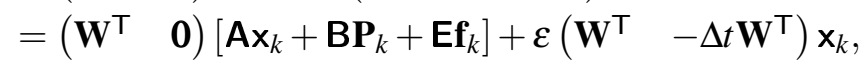

which can compactly be written as

$$
\boldsymbol{\xi}_{k}=\mathbf{C x}_{k}+\mathbf{D P}_{k}+\mathbf{F} \mathbf{f}_{k}
$$

with the corresponding matrices

$$
\mathbf{C}=\left(\begin{array}{c}
{\left[(1+\varepsilon) \mathbf{I}-\Delta t^{2} \mathbf{M}^{-1} \mathbf{K}\right]^{\top} \mathbf{W}} \\
\Delta t\left[(1-\varepsilon) \mathbf{I}-\Delta t \mathbf{M}^{-1} \mathbf{D}\right]^{\top} \mathbf{W}
\end{array}\right)^{\top}, \quad \mathbf{D}=\Delta t \mathbf{W}^{\top} \mathbf{M}^{-1} \mathbf{W}, \quad \mathbf{F}=\Delta t^{2} \mathbf{W}^{\top} \mathbf{M}^{-1} .
$$

The matrix $\mathbf{D}$ in (18) is a scaled version of the so-called Delassus matrix $\mathbf{W}^{\top} \mathbf{M}^{-1} \mathbf{W}$ [14], which is symmetric and positive definite as we assume $\mathbf{W}$ to have full column rank. In summary, the discrete system dynamics (14), (16) and (10) together with an output equation $\mathbf{y}_{k}=\mathbf{G x}_{k}$ (i.e. the available measurements) we have a discrete linear complementarity system of the form

$$
\begin{aligned}
\mathbf{x}_{k+1} & =\mathbf{A} \mathbf{x}_{k}+\mathbf{B} \mathbf{P}_{k}+\mathbf{E} \mathbf{f}_{k}, \\
\boldsymbol{\xi}_{k} & =\mathbf{C} \mathbf{x}_{k}+\mathbf{D} \mathbf{P}_{k}+\mathbf{F} \mathbf{f}_{k}, \\
0 & \leq \boldsymbol{\xi}_{k} \perp \mathbf{P}_{k} \geq 0, \\
\mathbf{y}_{k} & =\mathbf{G x}_{k},
\end{aligned}
$$

For a given $\mathbf{x}_{k}$ and $\mathbf{f}_{k}$, the equations (19b) and (19c) form together a linear complementarity problem (LCP) [15, 16], which has to be solved for $\boldsymbol{\xi}_{k}$ and $\mathbf{P}_{k}$ in each time step.

Remark 1. As noted in [11], the time-stepping scheme above admits a unique solution if the set $\mathscr{A}:=\left\{\mathbf{q} \in \mathbb{R}^{f} \mid \mathbf{g}(\mathbf{q}) \geq 0\right\}$ of admissible positions is convex and the excitation fulfills some regularity conditions. Here, we restrict ourselves to linear inequality constraints $\mathbf{g}(\mathbf{q})=\mathbf{W}^{\top} \mathbf{q}$. It is therefore straightforward to verify that $\mathscr{A}$ is always convex in our setting. Also, the LCP (19b), $(19 c)$ has a unique solution if all principal minors of the matrix $\mathbf{D}$ are strictly positive (i.e. it is a so-called $\mathscr{P}$-matrix, see [15]), which is fulfilled since $\mathbf{D}$ is symmetric and positive definite. 


\section{PASSIVITY-BASED OBSERVER}

For continuous-time linear complementarity systems, Heemels et al. [17] suggest a Luenbergertype state observer, where the observer gains are determined based on a linear matrix inequality. In the following, we show that an equivalent procedure is applicable for a discrete linear complementarity system of the form

$$
\begin{aligned}
\mathbf{x}_{k+1} & =\mathbf{A} \mathbf{x}_{k}+\mathbf{B} \mathbf{w}_{k}+\mathbf{E} \mathbf{v}_{k}, \\
\mathbf{z}_{k} & =\mathbf{C} \mathbf{x}_{k}+\mathbf{D} \mathbf{w}_{k}+\mathbf{F} \mathbf{v}_{k}, \\
0 & \leq \mathbf{z}_{k} \perp \mathbf{w}_{k} \geq 0, \\
\mathbf{y}_{k} & =\mathbf{G} \mathbf{x}_{k} .
\end{aligned}
$$

For a more standard notation, we denote the state by $\mathbf{x}_{k}$, the input by $\mathbf{v}_{k}$, the output by $\mathbf{y}_{k}$ and the complementary variables by $\mathbf{z}_{k}$ and $\mathbf{w}_{k}$, playing the role of the kinematic variable $\boldsymbol{\xi}_{k}$ and the discrete impulse $\mathbf{P}_{k}$ in (19).

The proposed Luenberger-type state observer for the discrete LCS (20) is in analogy to [17]

$$
\begin{aligned}
\hat{\mathbf{x}}_{k+1} & =\mathbf{A} \hat{\mathbf{x}}_{k}+\mathbf{B} \hat{\mathbf{w}}_{k}+\mathbf{E} \mathbf{v}_{k}+\mathbf{L}_{1}\left(\mathbf{y}_{k}-\hat{\mathbf{y}}_{k}\right), \\
\hat{\mathbf{z}}_{k} & =\mathbf{C} \hat{\mathbf{x}}_{k}+\mathbf{D} \hat{\mathbf{w}}_{k}+\mathbf{F} \mathbf{v}_{k}+\mathbf{L}_{2}\left(\mathbf{y}_{k}-\hat{\mathbf{y}}_{k}\right), \\
0 & \leq \hat{\mathbf{z}}_{k} \perp \hat{\mathbf{w}}_{k} \geq 0 \\
\hat{\mathbf{y}}_{k} & =\mathbf{G} \hat{\mathbf{x}}_{k},
\end{aligned}
$$

where all observer related variables are written with a circumflex $\left({ }^{\wedge}\right)$. The state observer contains two correction terms, both linear in the output difference (which is known through measurements). Defining the estimation errors as $\tilde{\mathbf{x}}_{k}:=\mathbf{x}_{k}-\hat{\mathbf{x}}_{k}, \tilde{\mathbf{z}}_{k}:=\mathbf{z}_{k}-\hat{\mathbf{z}}_{k}$ and $\tilde{\mathbf{w}}_{k}:=\mathbf{w}_{k}-\hat{\mathbf{w}}_{k}$, it follows that

$$
\begin{aligned}
\tilde{\mathbf{x}}_{k+1} & =\left(\mathbf{A}-\mathbf{L}_{1} \mathbf{G}\right) \tilde{\mathbf{x}}_{k}+\mathbf{B} \tilde{\mathbf{w}}_{k}, \\
\tilde{\mathbf{z}}_{k} & =\left(\mathbf{C}-\mathbf{L}_{2} \mathbf{G}\right) \tilde{\mathbf{x}}_{k}+\mathbf{D} \tilde{\mathbf{w}}_{k}, \\
\tilde{\mathbf{z}}_{k}^{\top} \tilde{\mathbf{w}}_{k} & \leq 0 .
\end{aligned}
$$

The last inequality in (22) expresses the maximal monotonicity of the discrete contact/impact law. It is easily checked by expanding

$$
\tilde{\mathbf{z}}_{k}^{\top} \tilde{\mathbf{w}}_{k}=\left(\mathbf{z}_{k}-\hat{\mathbf{z}}_{k}\right)^{\top}\left(\mathbf{w}_{k}-\hat{\mathbf{w}}_{k}\right)=\mathbf{z}_{k}^{\top} \mathbf{w}_{k}-\mathbf{z}_{k}^{\top} \hat{\mathbf{w}}_{k}-\hat{\mathbf{z}}_{k}^{\top} \mathbf{w}_{k}+\hat{\mathbf{z}}_{k}^{\top} \hat{\mathbf{w}}_{k} .
$$

Therein, the first and the last term vanish and the two other terms are non-positive due to the inequality complementarities in (20) and (21). The inequality $\tilde{\mathbf{z}}_{k}^{\top} \tilde{\mathbf{w}}_{k} \leq 0$ is however not an inequality complementarity. The equations (22) do therefore not form a full description of the error dynamics, because $\tilde{\mathbf{w}}_{k}$ cannot be expressed as a function of the estimation error $\tilde{\mathbf{x}}_{k}$. We rather have to use the last three lines of (20) and (21). As a consequence, $\tilde{\mathbf{w}}_{k}$ depends on $\mathbf{x}_{k}, \hat{\mathbf{x}}_{k}$ and $\mathbf{v}_{k}$, where $\hat{\mathbf{x}}_{k}$ can be replaced by $\mathbf{x}_{k}-\tilde{\mathbf{x}}_{k}$ (or the other way around). As pointed out in [17] for the continuous-time case, the error dynamics is therefore non-autonomous and has two states, $\tilde{\mathbf{x}}_{k}$ and $\mathbf{x}_{k}$ (or alternatively $\tilde{\mathbf{x}}_{k}$ and $\hat{\mathbf{x}}_{k}$ ). However, only the estimation error $\tilde{\mathbf{x}}_{k}$ has to tend to zero as $k$ increases.

Now, even though (22) is not a full description of the estimation error dynamics, it contains sufficient information for a Lyapunov stability analysis. Indeed, we can select a quadratic Lyapunov function candidate $V\left(\tilde{\mathbf{x}}_{k}\right)=\tilde{\mathbf{x}}_{k}^{\top} \mathbf{P} \tilde{\mathbf{x}}_{k}$ with $\mathbf{P}=\mathbf{P}^{\top}>0$ and calculate

$$
\begin{aligned}
V\left(\tilde{\mathbf{x}}_{k+1}\right)-V\left(\tilde{\mathbf{x}}_{k}\right) & =\tilde{\mathbf{x}}_{k+1}^{\top} \mathbf{P} \tilde{\mathbf{x}}_{k+1}-\tilde{\mathbf{x}}_{k}^{\top} \mathbf{P} \tilde{\mathbf{x}}_{k} \\
& =\left(\tilde{\mathbf{x}}_{k+1}+\tilde{\mathbf{x}}_{k}\right)^{\top} \mathbf{P}\left(\tilde{\mathbf{x}}_{k+1}-\tilde{\mathbf{x}}_{k}\right) \\
& =\left(\left(\mathbf{A}-\mathbf{L}_{1} \mathbf{G}\right) \tilde{\mathbf{x}}_{k}+\mathbf{B} \tilde{\mathbf{w}}_{k}+\tilde{\mathbf{x}}_{k}\right)^{\top} \mathbf{P}\left(\left(\mathbf{A}-\mathbf{L}_{1} \mathbf{G}\right) \tilde{\mathbf{x}}_{k}+\mathbf{B} \tilde{\mathbf{w}}_{k}-\tilde{\mathbf{x}}_{k}\right),
\end{aligned}
$$

which only contains the observer gains $\mathbf{L}_{1}$. After first subtracting and then again adding the term 
$2 \tilde{\mathbf{z}}_{k}^{\top} \tilde{\mathbf{w}}_{k},(24)$ can be written as

$$
\begin{aligned}
& V\left(\tilde{\mathbf{x}}_{k+1}\right)-V\left(\tilde{\mathbf{x}}_{k}\right)=\left(\begin{array}{c}
\tilde{\mathbf{x}}_{k} \\
\tilde{\mathbf{w}}_{k}
\end{array}\right)^{\top}\left(\begin{array}{cc}
\left(\mathbf{A}-\mathbf{L}_{1} \mathbf{G}\right)^{\top} \mathbf{P}\left(\mathbf{A}-\mathbf{L}_{1} \mathbf{G}\right)-\mathbf{P} & \left(\mathbf{A}-\mathbf{L}_{1} \mathbf{G}\right)^{\top} \mathbf{P B}-\left(\mathbf{C}-\mathbf{L}_{2} \mathbf{G}\right)^{\boldsymbol{\top}} \\
\mathbf{B}^{\top} \mathbf{P}\left(\mathbf{A}-\mathbf{L}_{1} \mathbf{G}\right)-\left(\mathbf{C}-\mathbf{L}_{2} \mathbf{G}\right) & \mathbf{B}^{\top} \mathbf{P B}-\left(\mathbf{D}+\mathbf{D}^{\top}\right)
\end{array}\right)\left(\begin{array}{c}
\tilde{\mathbf{x}}_{k} \\
\tilde{\mathbf{w}}_{k}
\end{array}\right) \\
& +2 \tilde{\mathbf{z}}_{k}^{\top} \tilde{\mathbf{w}}_{k}
\end{aligned}
$$

which now contains both observer gains $\mathbf{L}_{1}$ and $\mathbf{L}_{2}$. Because $\tilde{\mathbf{z}}_{k}^{\top} \tilde{\mathbf{w}}_{k} \leq 0$, it follows that we have $V\left(\tilde{\mathbf{x}}_{k+1}\right)-V\left(\tilde{\mathbf{x}}_{k}\right) \leq-\mu V\left(\tilde{\mathbf{x}}_{k}\right)$ if the matrix inequality

$$
\left(\begin{array}{cc}
\left(\mathbf{A}-\mathbf{L}_{1} \mathbf{G}\right)^{\top} \mathbf{P}\left(\mathbf{A}-\mathbf{L}_{1} \mathbf{G}\right)-\mathbf{P}+\mu \mathbf{P} & \left(\mathbf{A}-\mathbf{L}_{1} \mathbf{G}\right)^{\top} \mathbf{P B}-\left(\mathbf{C}-\mathbf{L}_{2} \mathbf{G}\right)^{\top} \\
\mathbf{B}^{\top} \mathbf{P}\left(\mathbf{A}-\mathbf{L}_{1} \mathbf{G}\right)-\left(\mathbf{C}-\mathbf{L}_{2} \mathbf{G}\right) & \mathbf{B}^{\top} \mathbf{P B}-\left(\mathbf{D}+\mathbf{D}^{\top}\right)
\end{array}\right) \leq 0,
$$

holds. This matrix inequality is nonlinear in the unknowns $\mathbf{L}_{1}, \mathbf{L}_{2}$ and $\mathbf{P}$. However, by introducing $\mathbf{S}:=\mathbf{P L}_{1}$ and applying the Schur complement lemma, it can be checked that (26) is equivalent to the linear matrix inequality (LMI)

$$
\left(\begin{array}{ccc}
-\mathbf{P}+\mu \mathbf{P} & -\left(\mathbf{C}-\mathbf{L}_{2} \mathbf{G}\right)^{\top} & \mathbf{A}^{\top} \mathbf{P}-\mathbf{G}^{\top} \mathbf{S}^{\top} \\
-\left(\mathbf{C}-\mathbf{L}_{2} \mathbf{G}\right) & -\left(\mathbf{D}+\mathbf{D}^{\top}\right) & \mathbf{B}^{\top} \mathbf{P} \\
\mathbf{P A}-\mathbf{S G} & \mathbf{P B} & -\mathbf{P}
\end{array}\right) \leq 0 .
$$

Since $\mathbf{P}$ is invertible, $\mathbf{L}_{1}$ can be recovered in a second step as $\mathbf{L}_{1}=\mathbf{P}^{-1} \mathbf{S}$.

Remark 2. The matrix inequality (26) is linked to a passivity condition: A linear time-invariant discrete-time system of the form

$$
\begin{aligned}
\mathbf{x}_{k+1} & =\mathbf{A} \mathbf{x}_{k}+\mathbf{B} \mathbf{w}_{k}, \\
\mathbf{y}_{k} & =\mathbf{C} \mathbf{x}_{k}+\mathbf{D} \mathbf{w}_{k},
\end{aligned}
$$

written in short as system $(\mathbf{A}, \mathbf{B}, \mathbf{C}, \mathbf{D})$, is said to be passive if there exists a nonnegative function $V: \mathbb{R}^{n} \rightarrow \mathbb{R}$ (called the storage function) with $V(\mathbf{0})=0$ such that

$$
V\left(\mathbf{x}_{k+1}\right)-V\left(\mathbf{x}_{k}\right) \leq \mathbf{y}_{k}^{\top} \mathbf{w}_{k}
$$

$\forall \mathbf{w}_{k}$ and $\forall k$.

It can be shown, that system (28) is passive if and only if if there exists a matrix $\mathbf{P}=\mathbf{P}^{\top} \geq 0$ such that the matrix inequality

$$
\left(\begin{array}{cc}
\mathbf{A}^{\top} \mathbf{P A}-\mathbf{P}+\mu \mathbf{P} & \mathbf{A}^{\top} \mathbf{P B}-\mathbf{C}^{\top} \\
\mathbf{B}^{\top} \mathbf{P A}-\mathbf{C} & \mathbf{B}^{\top} \mathbf{P B}-\left(\mathbf{D}+\mathbf{D}^{\top}\right)
\end{array}\right) \leq 0
$$

is fulfilled with $\mu=0$. As a stronger condition we call system (28) strictly passive, if there exists a matrix $\mathbf{P}=\mathbf{P}^{\top} \geq 0$ such that (30) holds for any $\mu>0$. In that case, it can be shown that the inequality (29) holds strictly.

\section{CONCLUSION}

In this work, we proposed to attack the state observer problem for linear mechanical systems subjected to unilateral constraints from a Nonsmooth Dynamics perspective. After approximating the continuous-time problem using the Schatzman-Paoli scheme, we have shown that the discretetime system forms a discrete linear complementarity system for which, in principle, the discrete adaptation of an existing passivity-based state observer can be applied. Thereby we achieved a first step towards a state observer design for unknown impact time instants. A next step would be to investigate under which conditions the linear matrix inequality, which is a sufficient condition for the asymptotic stability of the estimation error dynamics, admits a solution. Furthermore, it is necessary to analyze the behavior of the resulting observer gains as the time step $\Delta t$ tends to zero. 


\section{REFERENCES}

[1] Tanwani, A., Brogliato, B., Prieur, C.: Observer design for unilaterally constrained Lagrangian systems: A passivity-based approach. IEEE Transactions on Automatic Control 61(9) (2016) 2386-2401

[2] Menini, L., Tornambè, A.: Velocity observers for non-linear mechanical systems subject to non-smooth impacts. Automatica 38(12) (2002) 2169-2175

[3] Baumann, M., Leine, R.I.: A synchronization-based state observer for impact oscillators using only collision time information. International Journal of Robust and Nonlinear Control 26(12) (2016) 2542-2563

[4] Menini, L., Tornambè, A.: State immersion observers for mechanical systems with impacts. In: 2016 IEEE 55th Conference on Decision and Control (CDC). (Dec 2016) 7117-7122

[5] Kim, J., Cho, H., Shamsuarov, A., Shim, H., Seo, J.H.: State estimation strategy without jump detection for hybrid systems using gluing function. In: 53rd IEEE Conference on Decision and Control. (Dec 2014) 139-144

[6] Glocker, Ch.: Set-valued Force Laws, Dynamics of Non-smooth Systems. Volume 1 of Lecture Notes in Applied Mechanics. Springer-Verlag (2001)

[7] Glocker, Ch.: Energetic consistency conditions for standard impacts. Part I: Newton-type inequality impact laws and Kane's example. Multibody System Dynamics 29(1) (2013) 77117

[8] Leine, R.I., van de Wouw, N.: Stability and Convergence of Mechanical Systems with Unilateral Constraints. Lecture Notes in Applied and Computational Mechanics. Springer, Germany (2008)

[9] Moreau, J.J.: Unilateral contact and dry friction in finite freedom dynamics. In: Non-Smooth Mechanics and Applications. Springer-Verlag (1988) 1-82

[10] Paoli, L., Schatzman, M.: A Numerical Scheme for Impact Problems I: The OneDimensional Case. SIAM Journal on Numerical Analysis 40(2) (January 2002) 702 - 733

[11] Paoli, L.: Mathematical aspects of vibro-impact problems. In Leine, R.I., Acary, V., Brüls, O., eds.: Advanced Topics in Nonsmooth Dynamics: Transactions of the European Network for Nonsmooth Dynamics. Springer International Publishing, Cham (2018) 135-189

[12] Heemels, W., Schumacher, J., Weiland, S.: Linear complementarity systems. SIAM J. Appl. Math 60 (1997) 2000

[13] Heemels, W.P.M.H., Brogliato, B.: The complementarity class of hybrid dynamical systems. European Journal of Control 9(2-3) (2003) 322-360

[14] Brogliato, B.: Nonsmooth Mechanics. Models, Dynamics and Control. 3 edn. Communications and Control Engineering. Springer-Verlag, International Publishing (2016)

[15] Cottle, R.W., Pang, J.S., Stone, R.E.: The Linear Complementarity Problem. Classics in Applied Mathematics. Society for Industrial and Applied Mathematics (SIAM) (1992)

[16] Murty, K.: Linear Complementarity, Linear and Nonlinear Programming. Sigma Series in Applied Mathematics. Heldermann (1988)

[17] Heemels, W.P.M.H., Camlibel, M.K., Schumacher, J.M., Brogliato, B.: Observer-based control of linear complementarity systems. International Journal of Robust and Nonlinear Control 21(10) (2011) 1193-1218 